\title{
Development and analysis of a 50-year high-resolution daily gridded precipitation dataset over Spain (Spain02)
}

\author{
S. Herrera $^{(a)}{ }^{*}$ J.M. Gutiérrez ${ }^{(a)}$, R. Ancell ${ }^{(b)}$, M.R. Pons ${ }^{(b)}$, M.D. Frías ${ }^{(c)}$ and J. Fernández ${ }^{(c)}$ \\ ${ }^{(a)}$ Instituto de Física de Cantabria, CSIC-University of Cantabria, Santander, Spain. \\ ${ }^{(b)}$ Agencia Estatal de Meteorología (AEMET), Santander, Spain. \\ ${ }^{(c)}$ Dept. of Applied Mathematics and Computer Science. Universidad de Cantabria. Santander, Spain.
}

(Accepted in International Journal of Climatology, 15 October 2010, doi: 10.1002/joc.2256)

\begin{abstract}
In this paper we present a new publicly available high-resolution daily precipitation gridded dataset developed for peninsular Spain and the Balearic islands using 2756 quality-controlled stations (this dataset is referred to as Spain02). The grid has a regular $0.2^{\circ}$ (aprox. 20km) horizontal resolution and spans the period from 1950 to 2003. Different interpolation methods were tested using a cross-validation approach to compare the resulting interpolated values against station data: Kriging, Angular Distance Weighting, and Thin Plane Splines. Finally, the grid was produced applying the kriging method in a two-step process. First, the occurrence was interpolated using a binary kriging and, in a second step, the amounts were interpolated by applying ordinary kriging to the occurrence outcomes. This procedure is similar to the interpolation method used to generate the $E-O B S$ gridded data - the state-of-the-art publicly available high-resolution daily dataset for Europe - which was used in this work for comparison purposes.

Climatological statistics and extreme value indicators from the resulting grid were compared to those from the $25 \mathrm{~km} E-O B S$ dataset using the observed station records as a reference. Spain02 faithfully reproduces climatological features such as annual precipitation occurrence, accumulated amounts and variability whereas E-OBS has some deficiencies in the southern region. When focusing on upper percentiles and other indicators of extreme precipitation regimes, Spain02 accurately reproduces the amount and spatial distribution of the observed extreme indicators, whereas $E-O B S$ data present serious limitations over Spain due to the sparse data used in this region. Since extreme values are more sensitive to interpolation, the dense station coverage of this new data set was crucial to get an accurate reproduction of the extremes.
\end{abstract}

KEY WORDS: daily precipitation; gridded dataset; kriging; Spain

${ }^{*}$ Corresponding author address: Sixto Herrera, Instituto de Física de Cantabria, CSIC-University of Cantabria, Avenida de los Castros s/n, Santander, 39005 Spain.

E-mail: sixto.herrera@unican.es 


\section{Introduction}

The analysis and characterization of precipitation at regional scale requires data at high temporal and spatial resolution due to the abrupt variations of this variable in time and space. Even when dense station data are available, temporal gaps are usually present and the spatial coverage is not always uniform. Historical quality controlled gridded datasets play an increasingly important role in climate studies (Trenberth 1997; Goddard et al. 2001). Also, in recent years there has been an increasing demand for comprehensive regular high-resolution (both in time and space) gridded datasets from different sectors, including hydrology, agriculture and health. Gridded data are also of major importance for the validation of global and regional climate models (Osborn and Hulme 1997) and for the regional adaptation of global predictions or projections using statistical downscaling methods (see, e.g. Maurer and Hidalgo 2008).

During the last two decades different gridded products have been developed at a global scale using sound geostatistical methodologies such as kriging, angular distance weighting (ADW) or thin plate splines (TPS) (see Sen 2009; Chiles and Delfiner 1999, for more details on geostatistical methods). Most of the available global high-resolution gridded products, such as the GPCP (Adler et al. 2003, see also http://precip.gsfc.nasa.gov), the CRU datasets (http://www.cru.uea.ac.uk/cru/data), and the worldclim (Hijmans et al. 2005, see also http://www.worldclim.org) have been developed on a monthly basis with a typical resolution of $100 \mathrm{~km}$. However, regional analysis require higher resolution datasets both in space (tens of kilometers) and time (intra-daily or daily data), so that climatic differences across sub-regions can be identified and both mean and extreme climate conditions can be analyzed. In the last few years some daily gridded products have been developed at continental-scale from dense networks of daily rain gauge data (thousands of stations) in Europe (E-OBS dataset, Haylock et al. 2008), North America (Maurer et al. 2002), South America (Liebmann and Allured 2005), or Asia (Yatagai et al. 2008) with typical resolutions of $50 \mathrm{~km}$. These grids allow for a detailed analysis of the mean climate conditions at a continental scale. However, due to the inhomogeneous coverage in different countries it is still not clear whether these continental grids can also characterize the specific extreme climate conditions of the different regions within the covered area. Country-like scale grids have been already developed in the Alps (Frei and Schär 1998) or the United Kingdom (Perry and Hollis 2005).

In this paper we develop a country-scale daily regular $0.2^{\circ}$ (aprox. $20 \mathrm{~km}$ ) grid in Spain, for the period 1950-2003 considering a very dense network of 2756 quality-controlled stations (we refer to this dataset as Spain02). Among the different interpolation methods tested using cross-validation: kriging, angular distance weighting and thin plate splines, the first two exhibited the best overall performance with very similar results. The grid was produced applying binary kriging for occurrence and later ordinary kriging for the amounts of the occurrence outcomes. This process is similar to the interpolation method used for the E-OBS gridded data, which is the state-of-the-art publicly available high-resolution daily dataset for Europe (Haylock et al. 2008), with the exception that elevation is not considered as a co-variable in Spain02, since the dense network used in the present study appropriately represents the orography in this region. As we shall see later, the resulting grid reproduce satisfactorily the upper percentiles (P95, P99) as well as some commonly used indicators of extreme precipitation regimes. Thus, we conclude that Spain02 can accurately reproduce the amount and spatial distribution of the observed extreme indicators, whereas $E-O B S$ data presents serious limitations in order to be used for the analysis of extremes in this area (due to the sparse station network available over Spain to build this grid).

This paper is organized as follows. Section 2 describes the area of study and the available data. Section 3 presents the different gridding methods tested in the study, the cross-validation results and the final algorithm applied to build the grid. Section 4 analyzes the annual climatology of the resulting grid, comparing the results with the observations and with the E-OBS dataset. Section 5 focuses on the extremes, considering extreme percentiles and other indicators, as well as some case-studies corresponding to remarkable extreme events. Finally, some conclusions and final remarks are given in Sec. 6. 


\section{Area of Study and Data}

\section{a. Area of Study}

Spain is located in southwestern Europe spanning a region with complex orography influenced by both Atlantic and Mediterranean climates (see Fig. 1a). These factors yield a marked local climate variability with a significant North-South precipitation gradient (Rodríguez-Puebla et al. 1998, 2001; Esteban-Parra et al. 1998; Muñoz-Díaz and Rodrigo 2004). Broadly speaking, Spain can be divided into five homogeneous climatic precipitation regions (Muñoz-Díaz and Rodrigo 2004): 1) The North Atlantic coast shows large amounts of precipitation (from 900 to $2500 \mathrm{~mm} / \mathrm{year}$ ) with a remarkable climatological regularity, mainly due to the continuous arrival of Atlantic frontal systems. 2) The central part presents low precipitation amounts (less than $500 \mathrm{~mm} / \mathrm{year}$ ) with the exception of the Tajo river basin (with $900 \mathrm{~mm} / \mathrm{year}$ ) with wet and cold air intrusions from Atlantic frontal systems. 3) The eastern coast is characterized by low annual amount of precipitation (less than $700 \mathrm{~mm} /$ year) with a large variability due to severe events (24h precipitations larger than $200 \mathrm{~mm}$ ); this is also the case of the Ebro river basin (northeast) due to wet and warm air intrusions from Mediterranean Sea (García-Ortega et al. 2007). 4) The southeastern region is a dry desert-like area with precipitation amounts less than $100 \mathrm{~mm} /$ year, with the exception of Sierra Nevada mountains. 5) The southwestern region is influenced by Atlantic winds, with rainfall over $900 \mathrm{~mm} / \mathrm{year}$. Note that these regions can be identified in the yearly precipitation climatology shown in Figures 6a-c and 6d-f, corresponding respectively to the observed and gridded precipitation datasets described in this paper.

Due to the rich climate conditions of the Iberian peninsula this region has been used as an illustrative area in different studies dealing with circulation indices (Rodríguez-Puebla et al. 2001; Goodess and Jones 2002), statistical downscaling techniques (von Storch et al. 1993; Goodess and Palutikof 1996), etc. However, these works have been carried out considering a limited number of local stations, which may not be representative of the existing spatial variability, particularly in the case of precipitation. High resolution regular gridded precipitation datasets could offer an alternative for those studies allowing a spatially homogeneous analysis; however, in spite of the great effort devoted to this task in recent years, the quality of publicly available gridded products over Spain is quite limited due to the sparse station network available for those studies in this region (for instance, the mean inter-station distance in the E-OBS gridded dataset over Spain is much larger than the grid spacing). The limitations of such sparse interpolated grids have been pointed out in different studies (see, e.g. Fernández and Sáenz 2003). In the present work we present a new publicly available gridded product obtained from a dense network of thousands of stations over Spain described in the next section.

\section{b. Data and Quality Control}

The database used in this study comprises daily accumulated precipitation records for 2756 Spanish gauges, covering the Iberian peninsular area and the Balearic islands (see Figure 2a). These gauges were selected from a set of over 9000 stations supplied by the Spanish Meteteorological Agency (AEMET), having met different quality criteria. As a first filter, we selected those stations with at least 20 years of data (35\% of valid records) overall in the period 1950-2003 (this value was chosen as a compromise between considering all the available daily information or including only those gauges with long time series); this filter reduced the number of stations down to 4139. Then, we tested the resulting stations for homogeneity, both absolute and relative (see Peterson et al. 2008, and references therein). In particular we considered yearly data and applied the standard normal homogeneity test (SNHT) for a single break and the Alexandersson test (Alexandersson 1986) to the resulting series; in the later case the reference series for each target station was built as the average of the values of the five neighbouring stations with larger overlapping period (with a minimum of 15 -years) within a distance of $0.3^{\circ}$. Only those stations passing both tests at a $95 \%$ confidence level were used to build the gridded dataset, resulting a total of 2756 valid stations. The mean distance between neighbouring pairs of the selected stations was about $7 \mathrm{~km}\left(0.063^{\circ}\right)$, with a standard deviation of 

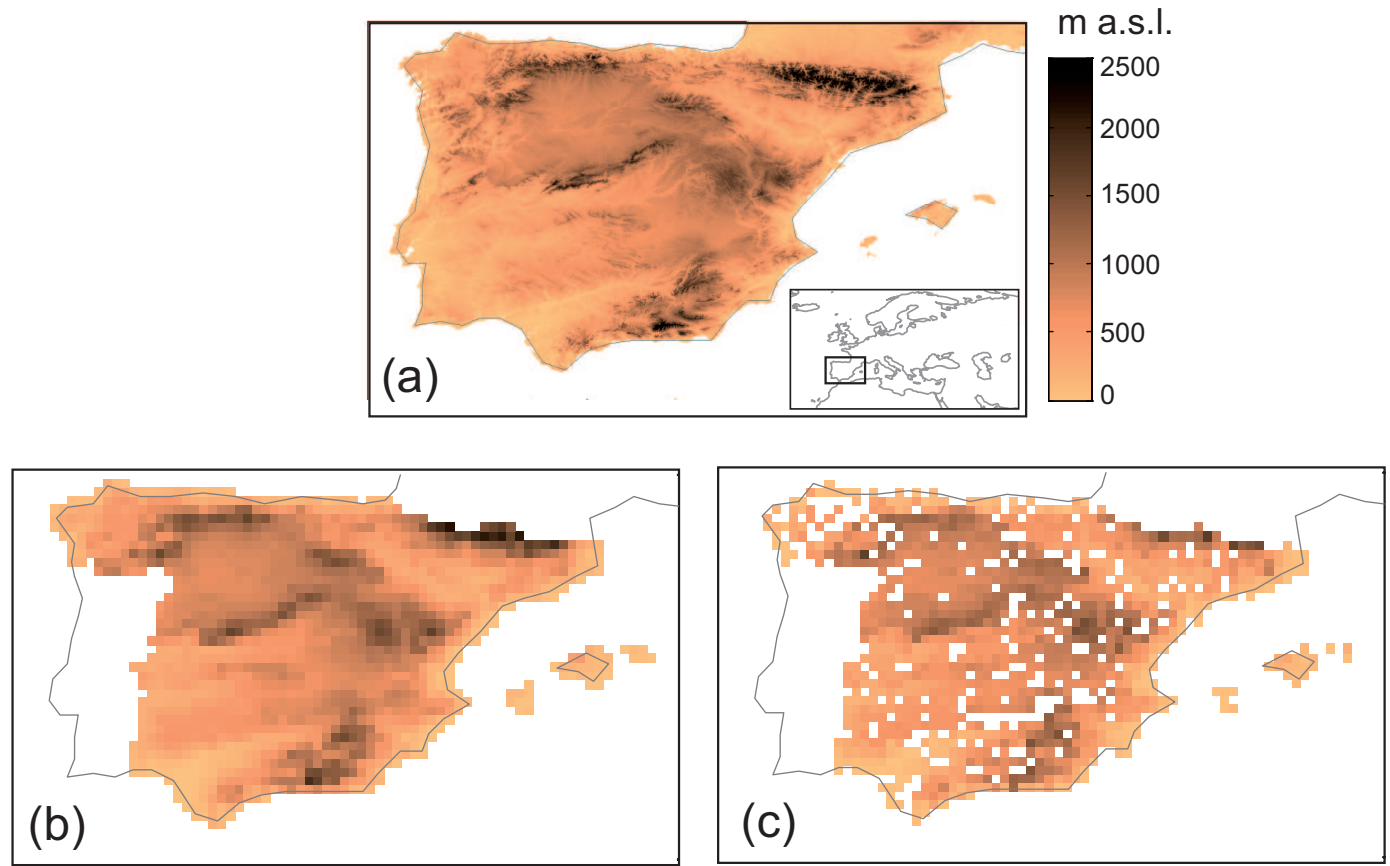

Figure 1: (a) Topography of the Iberian peninsula from GTOPO30 (approx. resolution $1 \mathrm{~km}$ ). (b) Topography interpolated to the regular $0.2^{\circ}$ grid used in SpainO2 dataset shown in Fig. $2 \mathrm{~b}$. (c) Effective altitude of the grid points, obtained as the weighted mean (according to temporal coverage) of the corresponding gauges elevation.

$0.032^{\circ}$ (the maximum distance is $28 \mathrm{~km}$ ). Thus, we considered a regular $0.2^{\circ}$ grid (see Fig. $2 \mathrm{~b}$ ) to interpolate the observations, as a compromise between spatial resolution and homogeneous coverage. Figure $2 \mathrm{c}$ shows the spatial coverage, given by the mean number of observations/stations daily available at each grid box in the period 1950-2003 (note that the number of empty grid boxes is only $15 \%$ of the total). Figure $2 \mathrm{~d}$ shows the temporal coverage, given by the mean percentage of daily records available in each grid box for the total period. These figures illustrate the dense and homogeneous coverage of the gauges used to build the grid.

Figure 3 shows the number of available stations year by year with different thresholds for the maximum allowed daily missing data (in \% units). This figure shows that the number of stations grows until 1970 where it remains approximately constant and later decreases in the last decade. Note that this poses limitations in order to use the gridded dataset for regional trend analysis (see Hofstra et al. 2009, for a detailed analysis of the influence of station density on the trends resulting in gridded data).

\section{Interpolation methodology}

Several geostatistical methods have been introduced in the literature to interpolate station data to a new arbitrary set of points (e.g. a regular grid) including kriging, angular distance weighting, thin plate splines, etc. (see Sen 2009; Chiles and Delfiner 1999, for more details on geostatistical methods). It has been shown that the performance of these methods varies with several factors, including the temporal and spatial scale and the density of stations (see, e.g. Hofstra et al. 2008). In this work we analyzed three popular gridding methods which have been previously tested and used in similar studies to produce daily precipitation gridded datasets. 

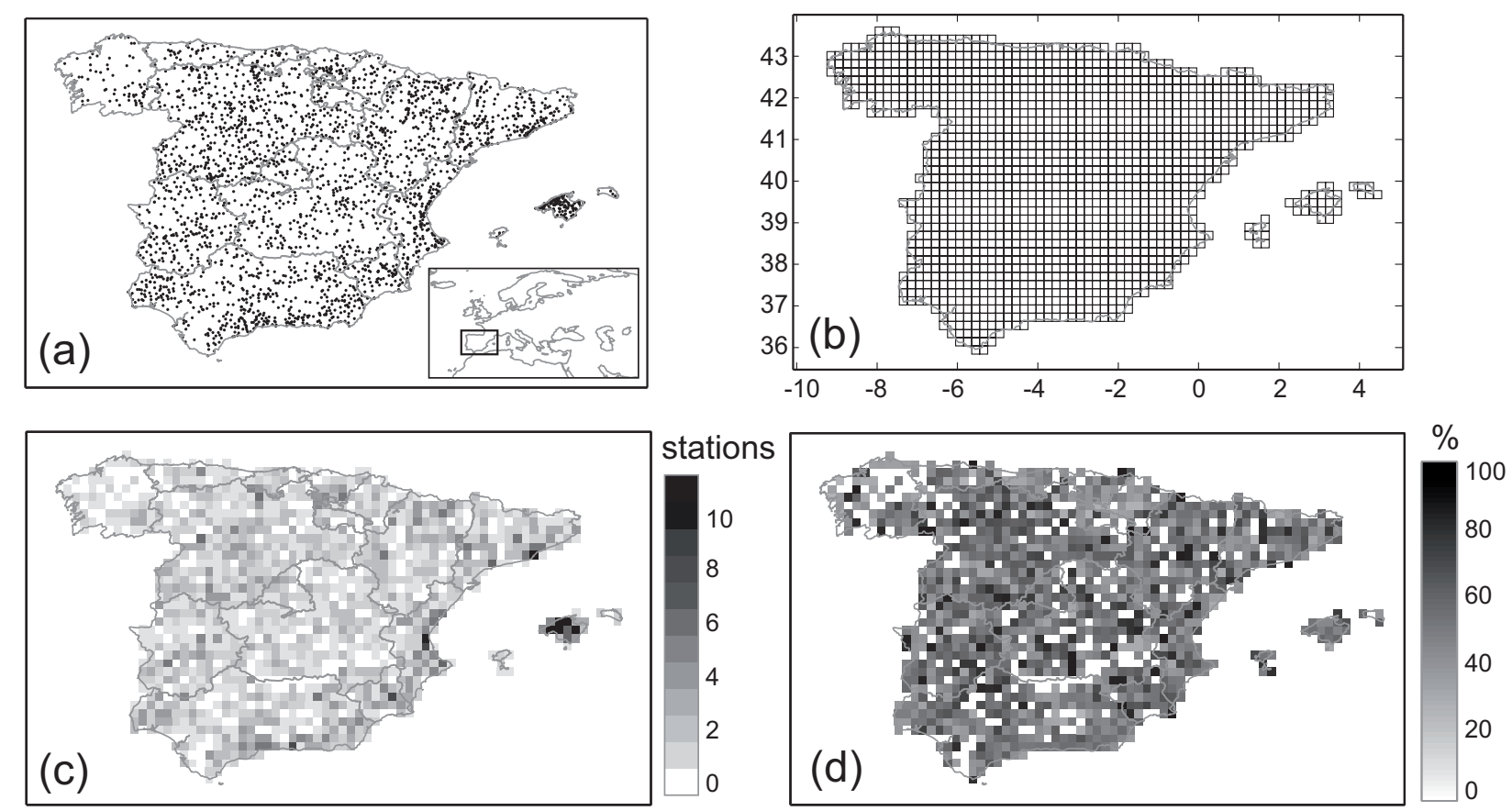

Figure 2: (a) Precipitation gauges selected to build the interpolated data set over the regular $0.2^{\circ}$ (aprox. 20km) grid shown in (b). (c) Grid stations density, obtained as the mean daily station records per grid point. (d) Temporal coverage (percentage of available records within the total period 1950-2003), obtained as the mean of the corresponding stations coverage per grid point.

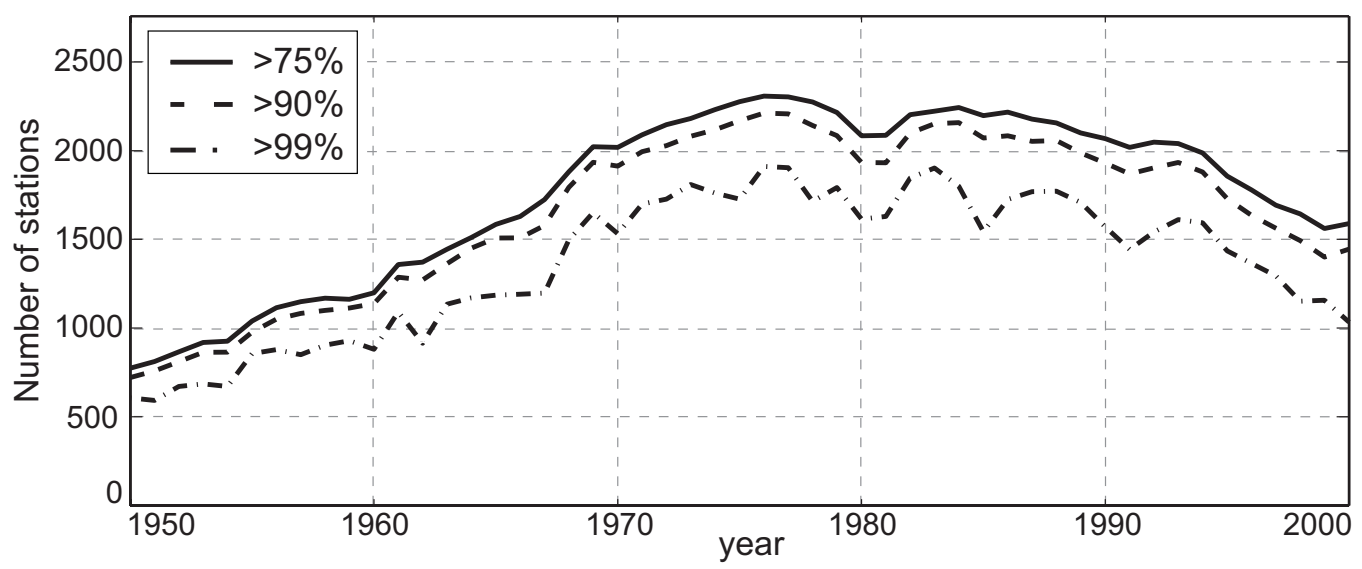

Figure 3: Temporal evolution of the number of available stations with at least 75, 90, and 99\% valid daily records within each particular year. 


\section{a. Gridding methods}

The kriging method is the most popular and widely used gridding methodology, with sound statistical basis (Matheron 1962; Biau et al. 1999). Kriging is a collection of linear regression methods that takes into account the stochastic nature of the data using a parametric variogram function to model the spatial correlation. Among these methods we applied ordinary kriging, assuming a constant term in the regression. We also considered an isotropic exponential family $\gamma(h)=r\left(1-e^{h / s}\right)$, for distances $h>0$, with range $r$ and sill $s$ parameters fitted to the data (note that we imposed a null nudget in the model). We tested different time windows to estimate the parameters obtaining optimal results with a unique global period (similar conclusions were also reported by Haylock et al. 2008). The globally estimated range parameter was $r=0.76$ and the sill $s=48.9$.

Angular distance weighting (Shepard 1968) is a weighting procedure using all the locations $\mathbf{x}_{1}, \ldots, \mathbf{x}_{k \equiv k(\mathbf{x})}$ ( $\mathbf{x}$ refers to the 2D longitude-latitude vector) with know values $y_{1}, \ldots, y_{k}$ within a radius $r$ of the target location $\mathbf{x}$ to produce an interpolated value $\hat{y}(\mathbf{x})=\sum_{i=1}^{k} w_{i} y_{i}$ with weights given by the two-stage composition of a distance component

$$
v_{i}=\left(e^{-d / c}\right)^{m},
$$

and an angular component

$$
w_{i}=v_{i}\left(1+\frac{\sum_{j \neq i} v_{j}(1-\cos (\Theta(j, i)))}{\sum_{j \neq i} v_{j}}\right)
$$

where $d$ is the distance between $\mathbf{x}$ and $\mathbf{x}_{i}, c$ is the correlation decay distance, $m$ is a constant exponent, and $\Theta(j, i)$ is the angle formed by $\mathbf{x}_{i}$ and $\mathbf{x}_{j}$ relative to the interpolation point $\mathbf{x}$. Although some evidence about the convenience of using anisotropic correlation decay has been recently found for Europe (Hofstra and New 2008), the variability is small in Spain, so we used an isotropic constant value for the interpolation process (this value was estimated from data as $d=0.15^{\circ}$ ). Moreover, the exponent value $m$ was set to 4 after cross-validation for optimal performance. A radius $r=0.5^{\circ}$ was considered to determine the local neighborhood of influence for each point.

Finally, thin plate splines were also used to produce a surface interpolated from data following the implementation by Hutchinson (1998a,b). This method can be though of as a particular non-parametric extension of multivariate regression, including a smooth non-parametric function determined from data in the following form:

$$
\hat{y}(\mathbf{x})=f(\mathbf{x})+\sum_{i=1}^{n} w_{i} \psi\left(\left\|\mathbf{x}-\mathbf{x}_{i}\right\|\right),
$$

where $\psi(r)=c r^{2} \log (r)$ is a radial basis function, $w_{i}$ are the weights to be estimated from data and $f(\mathbf{x})$ is a parametric linear sub-model including longitude and latitude as co-variables (note that other studies also consider elevation as a covariate in the linear sub-model). The parameters of the model are determined from data day by day by minimizing the predictive error resulting from (3), obtained implicitly as a cross validation error.

\section{b. Cross validation}

In order to assess the performance of the different interpolation methods, a cross-validation approach was carried out considering spatially stratified test samples. To this aim a $0.5^{\circ}$ regular grid was defined over the domain (with a total of 203 grid boxes) and a station in each box was randomly chosen and removed from the data (to be used later as a test) before applying the three interpolation algorithms to obtain the $0.2^{\circ}$ gridded series. Then, error scores were computed for every test station by comparing the observed values with the corresponding gridded one. In particular we used the bias, the root-mean-square error $(R M S E)$ and the Pearson correlation coefficient $R$ as validation measures; both the $R M S E$ and $R$ scores were computed 
on the subset of wet days (Precip $>0.1 \mathrm{~mm}$ ) in order to reduce the effects of the varying occurrence frequencies (see Fig. 6a). The random selection process for the test stations was repeated 10 times and mean scores across the 10 realizations were considered for each of the 203 validation boxes in the validation process.

Figure 4 compares the results of the ordinary kriging, ADW and TPS algorithms. Kriging method was selected as the reference and the other two methods are compared against kriging in this figure using scatterplot diagrams (each dot corresponds to one of the 203 validation grid boxes). The first column shows the mean daily bias for the whole period, whereas the second and third columns show the RMSE and the correlation coefficient $R$ for the wet days, respectively. These figures evidence a poor performance of the TPS method and a similar performance of kriging and ADW, with slightly better results for the former one. Therefore, we selected this sound geostatistical method to produce the daily gridded dataset.
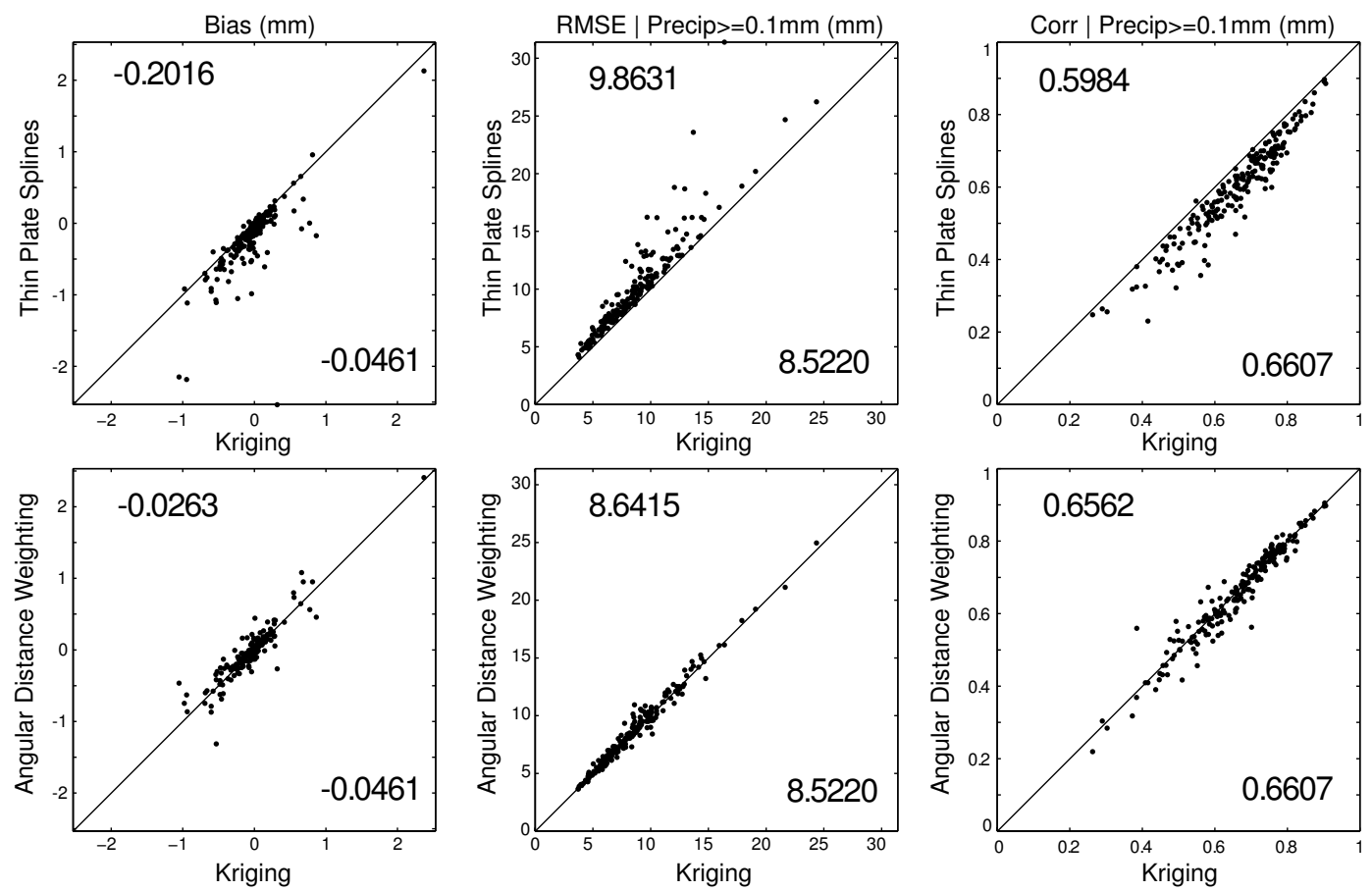

Figure 4: Scatter plots comparing TPS vs kriging (first row) and ADW vs kriging (second row) using three different statistical indices computed for each of the 203 grid boxes used in the crossvalidation experiment (see text for details). The indices are the bias (in mm), the RMSE (in mm) and correlation; the last two scores are computed considering only the subset of wet days (precipitation larger than $0.1 \mathrm{~mm}$ ). The numbers along the $x$ and $y$ axis indicate the mean values for the 203 validation points for the corresponding method.

Figure 5 shows the spatial distribution of the above scores for the kriging method over Spain for the daily data and the 5-day, weekly and monthly aggregated values (in different rows). This figure shows a very good agreement between the interpolated and observed series for aggregation times over five days with correlations larger than 0.85 in most of the grid points. However, the correlation for daily values is larger than 0.65 in most of the grid points, with bigger errors in regions with complex topography or larger variability. Thus, at daily timescale the interpolated value must be understood as an spatial average and does not correspond day by day with a particular station. 


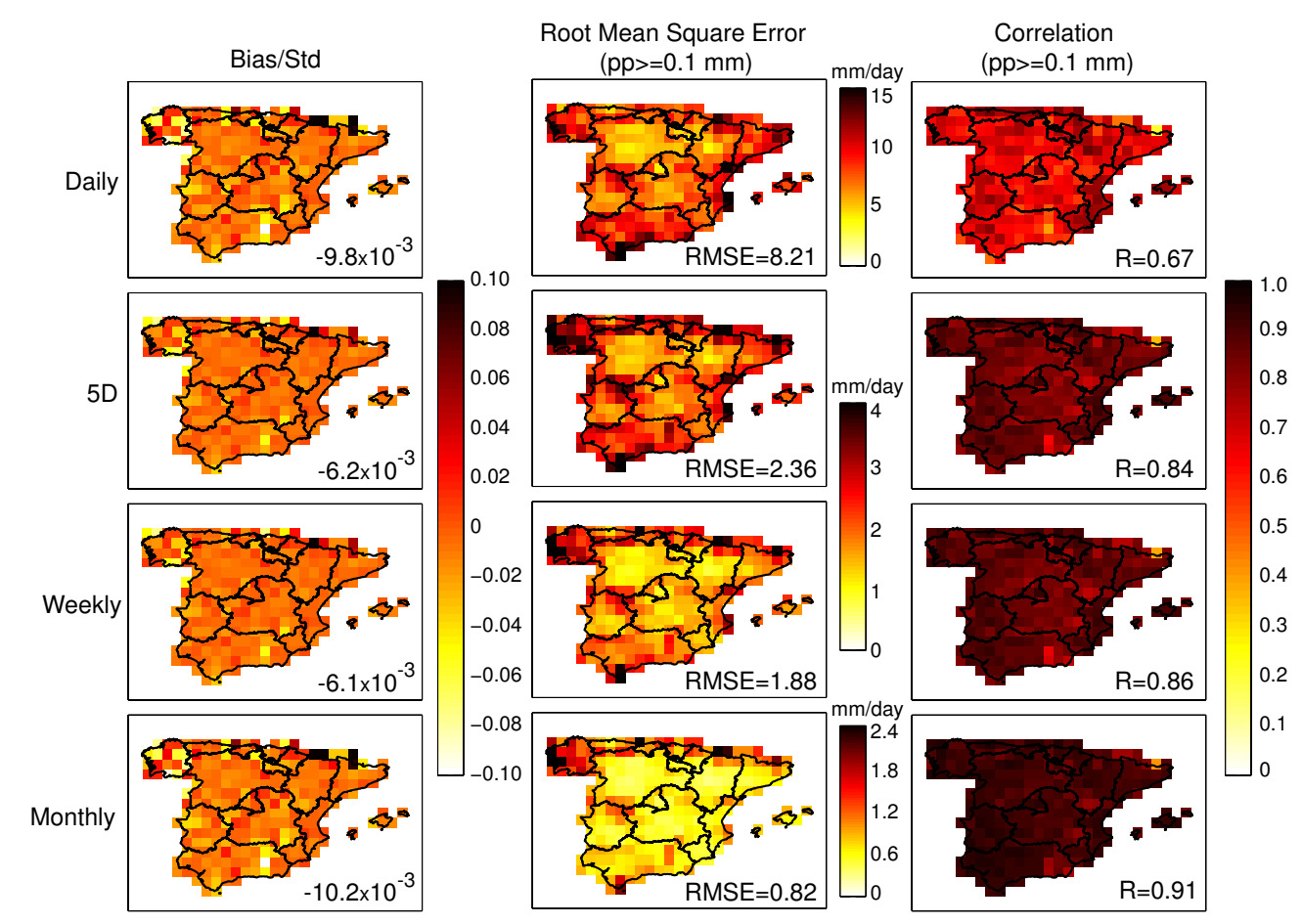

Figure 5: Maps of the mean scores associated to each of the grid boxes used in the cross validation (see text for details) for the gridded data set built using Kriging. The scores are the standardized bias of the full series and the RMSE and Pearson correlation coefficient $R$ of the wet days (precip $>0.1 \mathrm{~mm}$ ) for the original daily data (first row), and different temporal aggregations: 5day, weekly and monthly (in different rows). The spatial mean of each score is indicated on the lower-right corner of each panel.

\section{c. Interpolation Procedure}

The interpolation process on the $0.2^{\circ}$ grid was performed following a two-step indicator-ordinary kriging approach which allows reducing the smoothing effects around zero value zones. First, the occurrence of rainfall ( 0 or 1 depending on whether precip $>0.1 \mathrm{~mm}$ ) was interpolated applying indicator kriging considering a threshold of 0.5 for assigning a wet day to a grid point; indicator kriging makes no assumption of normality and is essentially a non-parametric counterpart to ordinary kriging (see Journel 1983; Deutsch and Journel 1998, for more details). In a second step, the amounts for wet days were interpolated by ordinary kriging as mentioned in the previous section. In this case, in order to account for the variable temporal density of stations (see Fig. 3) we went one step further and optimized the range and sill parameters decade by decade. The range parameter ranged from 0.64 to 0.87 (50.3 to 59.8 for the sill) in the different decades.

This process is similar to the interpolation approach followed in the E-OBS grid for Europe (developed in the ENSEMBLES project), with the exception that universal kriging was applied in this case, and a previous spline-based interpolation of monthly means was computed including elevation as a co-variable; thus, kriging was applied to the residuals. We decided not to explicitly include elevation in the development of Spain02 because the dense gauge network available in this study appropriately represents the orography corresponding to the $0.2^{\circ}$ grid. Figure $1 \mathrm{~b}$ and $\mathrm{c}$ show the orography corresponding to the $0.2^{\circ}$ grid interpolated from GTOPO30 and the effective orography of the grid, obtained as the weighted mean of the corresponding gauge elevations, respectively. This figure shows that the available gauges define a realistic orography of the region under study. 


\section{Analysis of Annual and Seasonal Precipitation}

In this section we analyze the capability of the gridded dataset to reproduce the precipitation climatology and seasonality of the observed data. Moreover, in order to compare this new dataset with other existing gridded data over the area, we considered the E-OBS daily grid which covers Europe at a $25 \mathrm{~km}$ resolution (Haylock et al. 2008). This is the best publicly available gridded product for Europe, although the station coverage is not homogeneous in space due to data availability/sharing limitations. In particular, in Spain only a few tens of stations were available to build the E-OBS grid. As we already mentioned, this inhomogeneity was partially compensated by including elevation in the gridding process. However, the different density of stations used in both grids must be taken into account when analyzing the results, since this is the main driving factor of the grid performance.

Figure 6 shows the daily rainfall frequency (annual occurrence, in percentage units), the annual accumulated amount (in $\mathrm{mm}$ ), and the daily variability (standard deviation of wet days, in $\mathrm{mm}$ ) for the observations and the Spain02 and E-OBS grids, in different rows. This figure shows that both grids appropriately capture the North-South gradient of the precipitation frequency and amount; however, E-OBS exhibits smoother patterns missing some of the regional information, specially in the central and southern regions. In the case of rainfall variability, on the one hand, E-OBS misses the regions with maximum values in the Mediterranean and South exhibiting a smooth pattern for the whole domain with values below $10 \mathrm{~mm}$ for all regions except Galicia (in the Northwestern). This lack of variability has been previously identified in other gridded datasets on sparse data regions (Fernández and Sáenz 2003). As we shall see later, this underestimation of the daily variability poses serious limitations in order to analyze high percentile values or extreme indicators. On the other hand, Spain02 appropriately represents the spatial variability and therefore, allows characterizing the different climatic regions described in Sec. 2.

Figure 7 compares the accumulated seasonal climatologies. This figures show how both gridded datasets appropriately capture the seasonal cycle, with E-OBS showing again a smoother pattern and lacking some centers of precipitation as expected due to the lack of spatial coverage in those regions.

\section{Analysis of Extremes}

One of the main shortcomings of gridded datasets is that extreme events can be smoothed during the interpolation process; note that heavy rainfall events can be very local and, hence, interpolation with neighboring stations may lead to an underestimation of the event amount. In this section we analyze both upper percentiles and extreme indicators commonly used to characterize extreme precipitation regimes. Moreover, we also show the performance of the gridded dataset to capture both the intensity and the spatial structure of severe precipitation episodes which constitute characteristic ephemerides of extreme weather in the Iberian peninsula.

\section{a. Upper percentiles}

Upper percentiles are often used as indicators of extreme precipitation, since they are obtained from the upper tail of the distribution. Figure 8 shows the 90, 95 and 99 percentiles of the observations and the gridded datasets. This figure shows that Spain02 appropriately characterize all these percentiles, both the spatial distribution and the amounts. Thus, it seems that the density of stations and grid spacing allow for a robust interpolation process, with apparently no smoothing of the extremes. Note that, as mentioned before, the situation for the $E-O B S$ grid is different. Although the spatial regions with higher percentile values are broadly recognized by this data set, the overall pattern is smoothed and some important areas suffering from extreme rainfall events (e.g. Valencia, on the Mediterranean coast) are missing. 


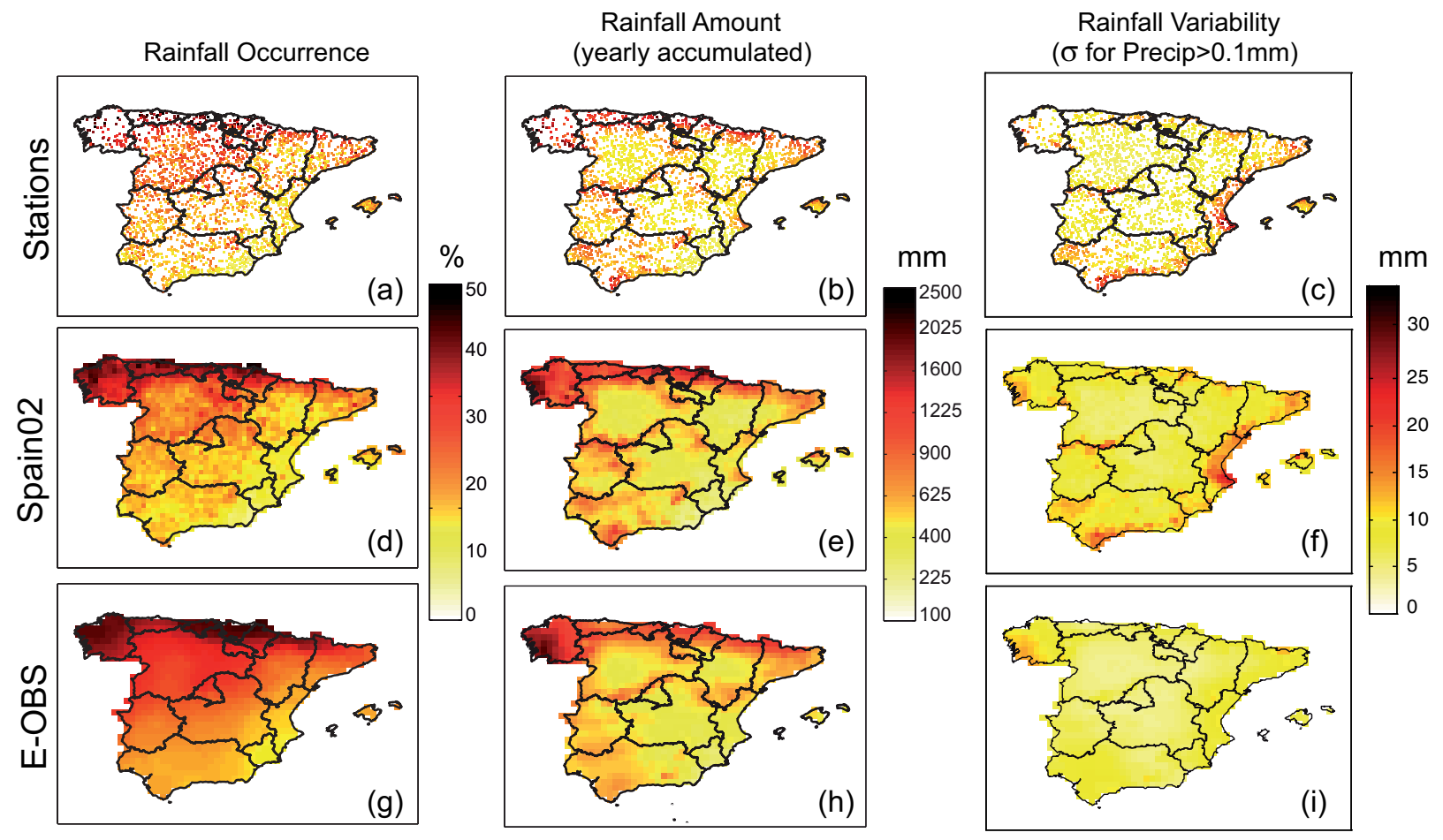

Figure 6: Rainfall frequency (annual occurrence, in percentage units), annual accumulated amount (in squared root scale with labels in the original units, for a better contrast of spatial differences), and the variability (standard deviation of wet days) for the observations (a)-(c) the Spain02 grid (d)-(f) and the E-OBS grid (g)-(i). 

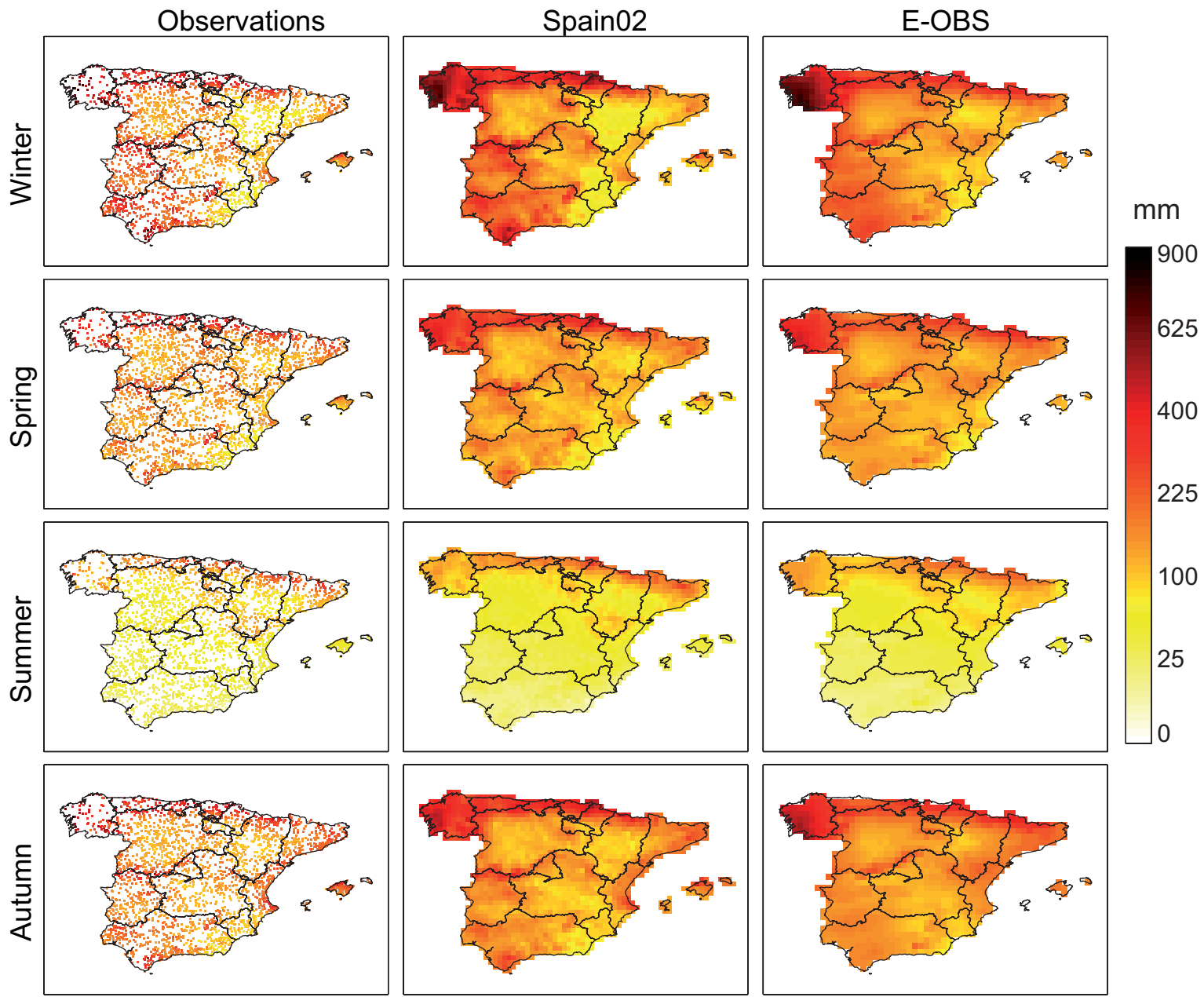

Figure 7: Seasonal accumulated precipitation (square-root scale with labels in the original units) derived from the observations and the Spain02 and E-OBS grids. 

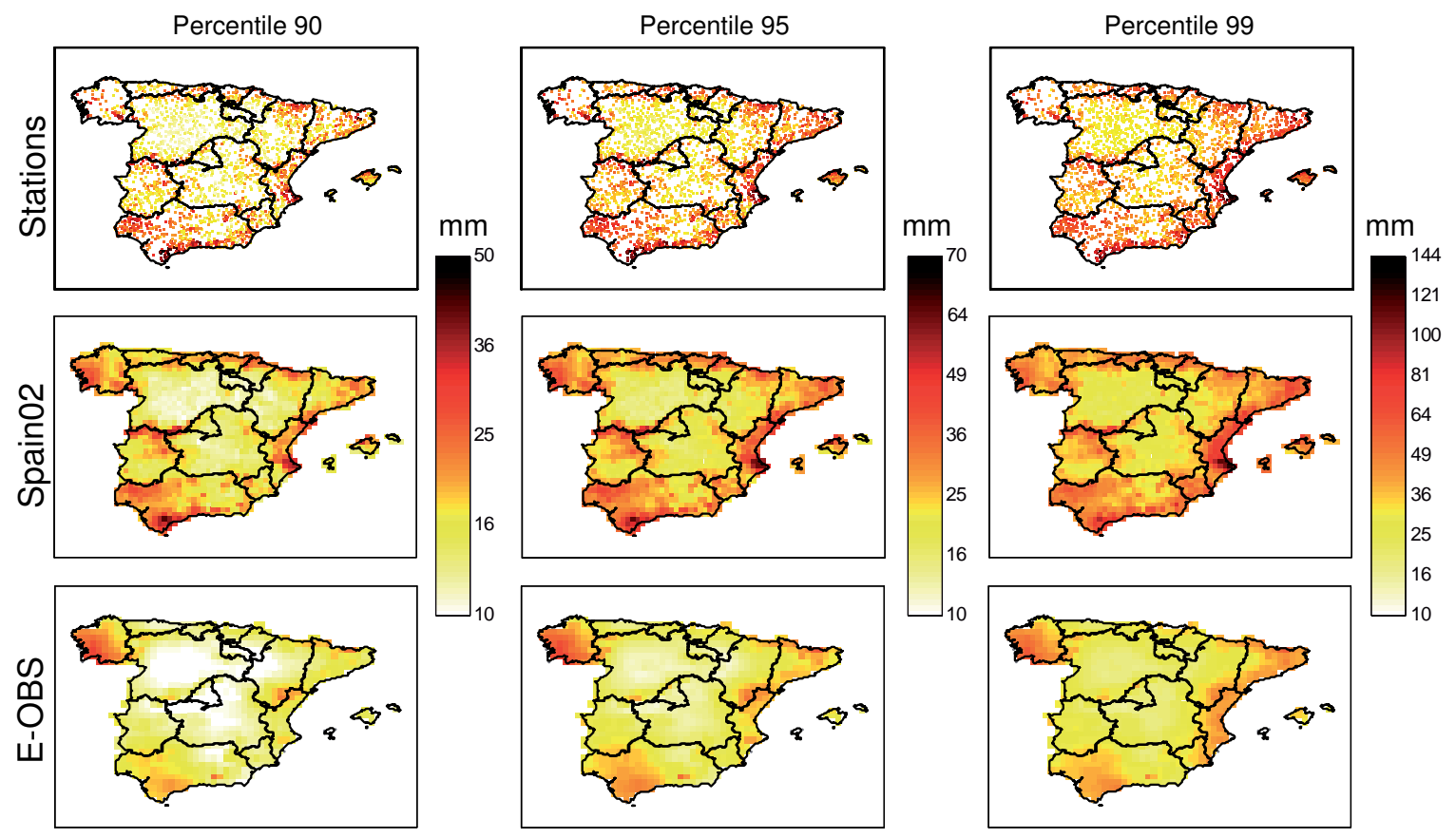

Figure 8: Percentiles 90, 95 and 99 (in squared root scale with labels in the original units) for the observations, the Spain02 grid and the E-OBS grid.

\section{b. Indicators of extreme precipitation}

We also analyzed some standard statistical indicators of extreme precipitation and compare the results for the observed and interpolated datasets. The indicators considered are a subset of the joint CCl/CLIVAR/JCOMM Expert Team on Climate Change Detection and Indices (ETCCDI) shown in Table 1 (see Sillmann and Roeckner 2008, for more details).

Table 1: Indicators of extreme precipitation defined by ETCCDI used in this study, http://cccma.seos.uvic.ca/ETCCDI. ( *) Note that the original R95p indicator has been modified considering its contribution to the total precipitation.

\begin{tabular}{lll} 
ID & Indicator & Units \\
\hline CDD & Annual maximum consecutive dry days $($ precip $<1 \mathrm{~mm})$ & days \\
CWD & Annual maximum consecutive wet days $($ precip $\geq 1 \mathrm{~mm})$ & days \\
R10 & Annual number of heavy precipitation days $($ precip $\geq 10 \mathrm{~mm})$ & days \\
R20 & Annual number of very heavy precipitation days $($ precip $\geq 20 \mathrm{~mm})$ & days \\
R95p/TOT & Percentage of total precipitation contributed by 5\% most rainy days & $\%$ \\
RX1day & Max 1-day precipitation amount & $\mathrm{mm}$ \\
RX5day & Max 5-day precipitation amount & $\mathrm{mm}$ \\
\hline
\end{tabular}

Figure 9 shows the spatial patterns of the first four indicators shown in Table 1, corresponding to the mean dry and wet spells (mean of maximum yearly values) and the absolute annual frequencies of heavy and very-heavy precipitation days (mean of yearly frequency values), respectively. On the one hand, both grids overestimate the dry and wet spells, specially in the southwestern peninsula. On the other hand, the 
results of Spain 02 for $R 10$ and $R 20$ are very good, whereas $E$-OBS underestimates the frequency of heavy precipitation specially in the Central and Southern regions, and also in the North for $R 20$.
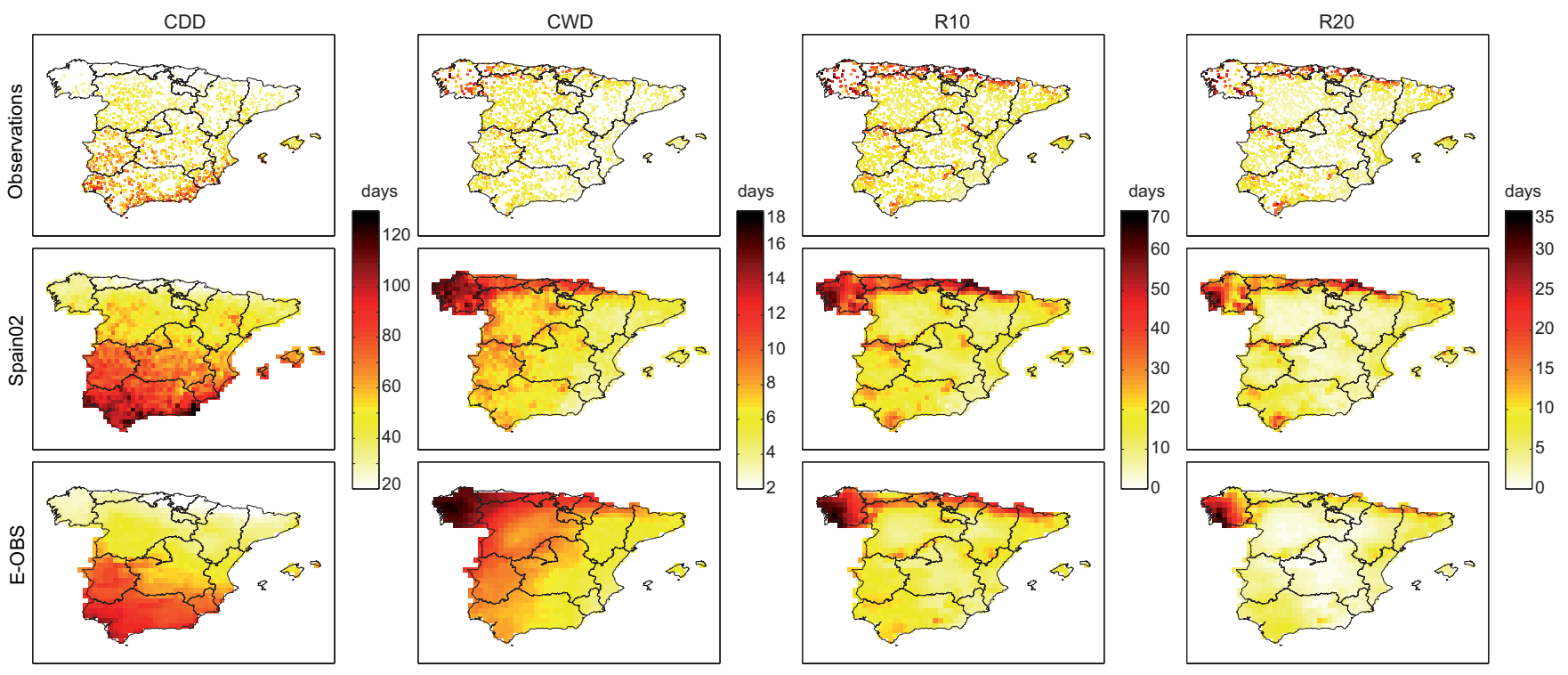

Figure 9: Values of the indicators $C D D, C W D, R 10$ and $R 20$ (see Table 1) for the observations and the gridded datasets Spain02 and E-OBS (in different rows). Means of annual values over the whole period are represented.

Figure 10 shows the spatial patterns of the last three indicators shown in Table 1, corresponding to the contribution of extreme precipitation (5\% most rainy days) to the total precipitation and the maximum 1 and 5-day accumulated precipitations; in all cases, averages of annual values for the whole period are shown. Note the good agreement between the observations and the Spain02 interpolated values, suggesting the suitability of this gridded dataset to characterize and study extreme precipitation in Spain. On the contrary, $E-O B S$ dataset should not be used for this purpose due to the underestimation found for these indicators.

\section{c. Case studies: Severe daily events}

In this section we focus on some case studies corresponding to characteristic severe precipitation events registered in the observed data over the Iberian peninsula. We analyze the temporal and spatial evolution of particular episodes (ephemerides) as a validation of the gridded data to represent the evolution of these extreme events. In particular we describe in detail the daily precipitation maximum that took place on November 3-4th 1987 in the Mediterranean coast due to a cut off low situation. This episode is registered as the record daily maximum precipitation in the last century in Spain achieving values around $800 \mathrm{~mm}$ in some stations over the Valencia area. Figure 11 shows the daily accumulated precipitation for the observations and the Spain02 and E-OBS grids corresponding to the period 2 to 5 November 1987. Spain02 preserves the high precipitation values observed in the Southeastern Mediterranean coast and also the spatial and temporal evolution of this event. This event is not properly detected by the E-OBS grid neither in time nor in intensity, showing smoothed values over the Valencia area. In fact, according to E-OBS, this event was much less extreme and the main center is displaced south to the Murcia region. The lack of stations during this period on the area makes the extreme event disappear or appear less intense and displaced (due to capturing only a small part of the precipitation pattern).

The analysis of some other particular ephemerides observed over Spain (not shown here) illustrates the capability of Spain02 for studying severe precipitation events. The conclusions for those other situations 

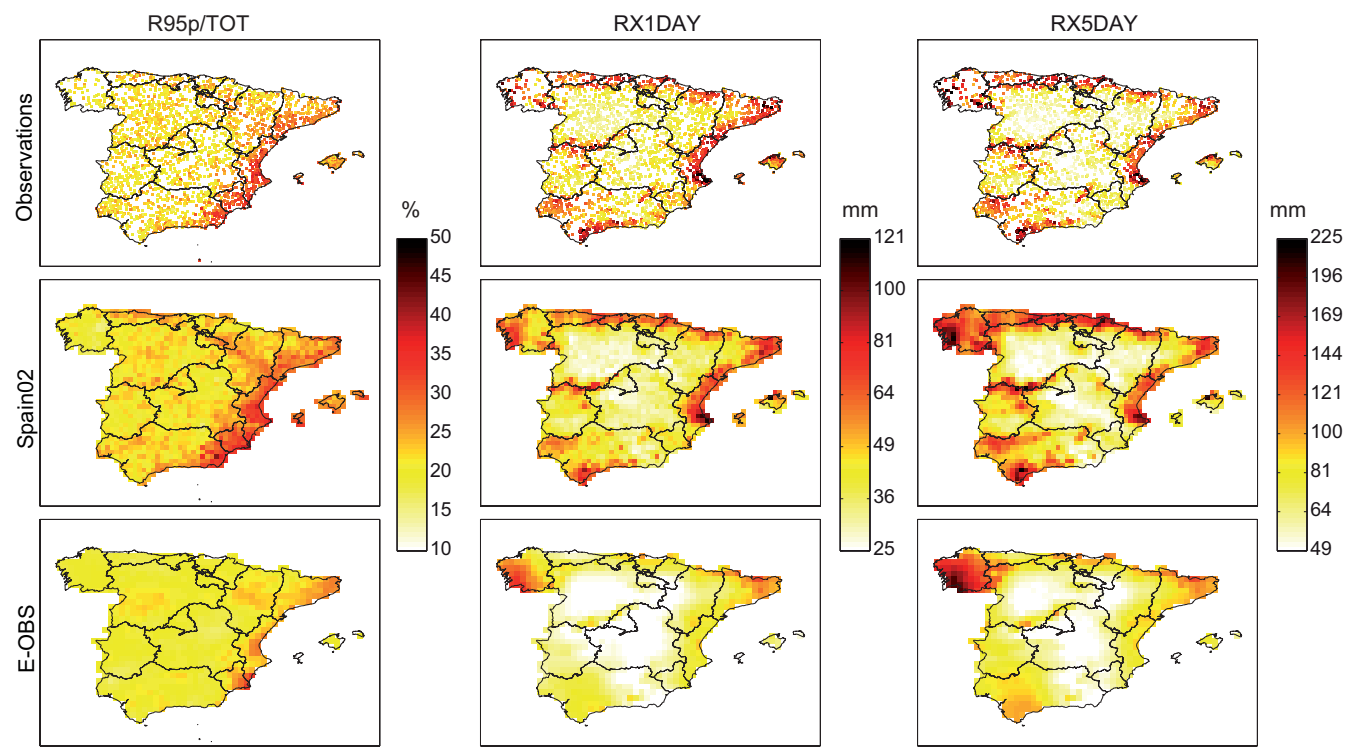

Figure 10: As Fig. 9, but for $R 95 p / T O T, R X 1 D A Y$ and $R X 5 D A Y$. For a better contrast of spatial differences, the last two indices are shown in squared root scale with labels in the original units.
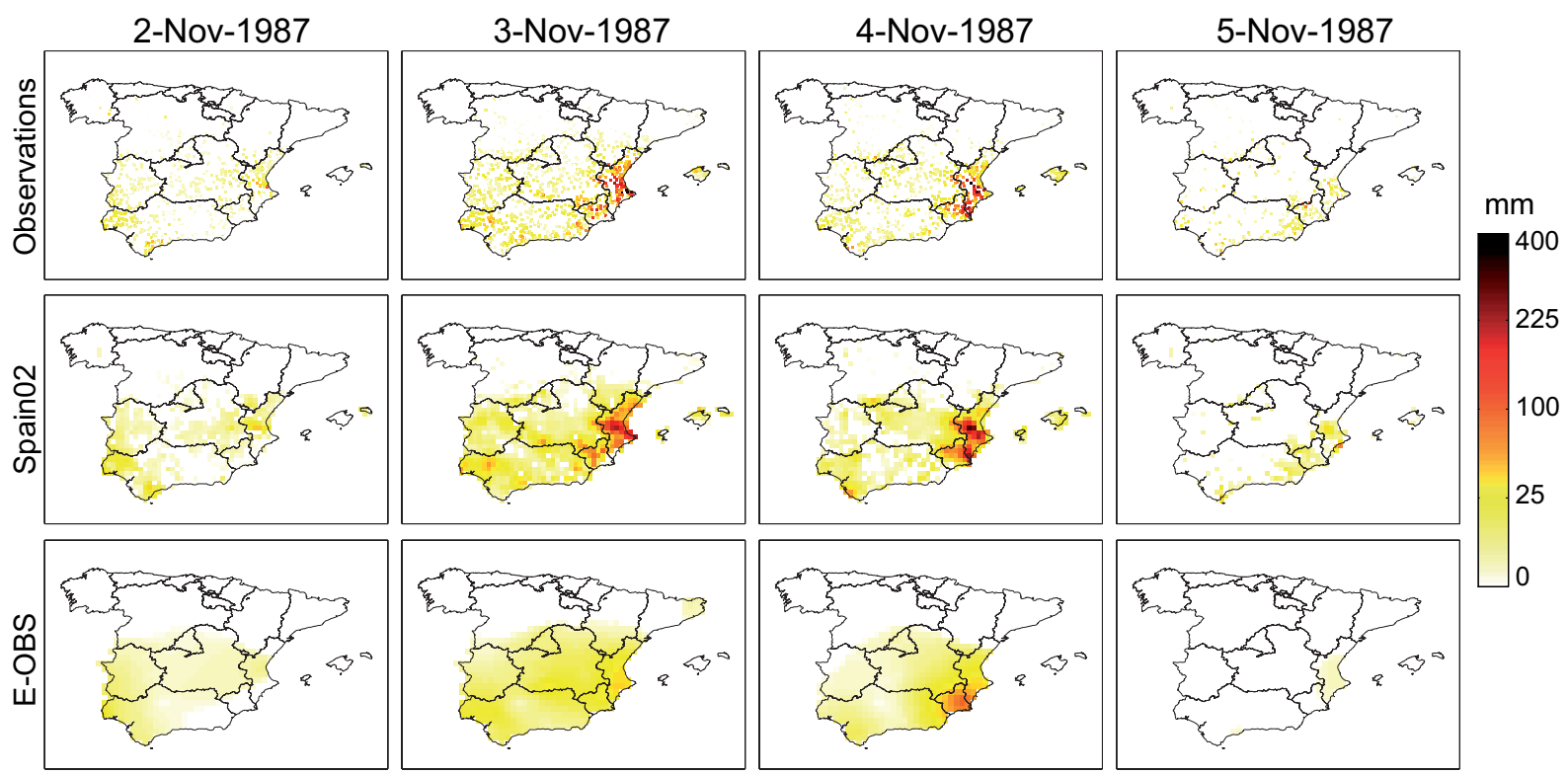

Figure 11: Daily accumulated precipitation (in square-root scale) for the observations and the Spain02 and E-OBS grids corresponding to the period 2 to 5 November 1987. 
are similar to the particular event shown here. More complete information about these severe precipitation events can be found at http://www. meteo. unican.es/spain02. As already found in Sections 5a and $b$, the ephemerides study also reveals that the E-OBS dataset underestimates the precipitation in extreme events. The lack of stations available in Spain to build E-OBS limits the capability of this grid to perform studies focused on extreme events in this area.

\section{Conclusions}

A new high-resolution daily precipitation gridded dataset for peninsular Spain and the Balearic islands is presented (Spain02). A dense number of quality-controlled stations was selected to develop the Spain02 grid with a regular $0.2^{\circ}$ horizontal resolution covering the period from 1950 to 2003. This new product is publicly available for non-commercial purposes.

The gridding methodology used is based on kriging since this approach showed the best performance among the two other interpolation methods considered (angular distance weighting and thin plane splines). The resulting dataset shows good cross-validation scores on a daily basis and these are much improved when considering 5-day, weekly or monthly accumulated data.

Spain02 mean and extreme value indicators were compared to the $25 \mathrm{Km}$ E-OBS grid developed in the ENSEMBLES project, which is the best daily dataset for the whole Europe to date. Using the observed station records as reference, both grids captured the observed climatological features. However E-OBS exhibits smoother patterns missing some of the regional information. Moreover, E-OBS misses most of the rainfall variability. The analysis of extreme value indicators shows the capability of the Spain02 grid to reproduce the intensity and spatial variability of the typical observed extreme indices whereas E-OBS underestimates those values in regions suffering of extreme precipitation events. These results highlight the main shortcoming of gridded datasets to perform studies on extreme events. Both Spain02 and E-OBS grids have been built using similar methodologies and, as shown in this study, properly reproduce the climatology of the region. However, the different number of stations available in Spain for the gridding process (2756 in Spain02 and a few tens in E-OBS) clearly limits the E-OBS capability to capture extreme precipitation episodes. This limitation was also shown in the analysis of several ephemerides where the Spain02 captures the spatial an temporal evolution of these events whereas the E-OBS grid smooths or does not detect the high precipitation values. Note that the analysis of extreme events is an active area of research in connection with climate change studies and the high quality and resolution of Spain02 makes this dataset an appropriate product for analyzing these events.

It is necessary to stress that the results shown apply only to Spain, a region with a specially poor station coverage in the E-OBS grid. E-OBS has much better coverage in other regions of Europe and is currently the best daily gridded data set for pan-European studies.

A cautionary remark must also be made concerning the variability of the station density in time (see Fig. 3 ), which poses serious limitations in order to use the Spain02 gridded dataset for regional trend analysis. This grid was developed with the focus on including as many trustworthy data as possible for each day. The results shown, reveal that the dataset retains high resolution information on extremes and can reproduce the day by day evolution of precipitation. However, in order to analyze trends, a reduced set of gauges with long records should be considered to build the grid. Such a grid would lose the high spatial resolution of Spain02 and is out of the scope of this work. We plan to produce such a gridded dataset (focused on trend analysis) in the future.

Public data distribution is an important issue in climate studies and it is one of the main contributions of this work. In order to allow researchers access this dataset, the Spanish Meteorological Agency (Agencia Estatal de Meteorología, AEMET) has made Spain02 publicly available for non-commercial purposes (see www.meteo.unican.es/spain02 for details). Thus, this product will allow a variety of detailed climate studies over Spain using a spatially homogeneous precipitation dataset. 
Acknowledgement The authors are grateful to AEMET for providing the necessary data to do this work and for distributing Spain02 freely for non-commercial purposes (see www. meteo. unican.es / spain 02 for details). We also acknowledge Spanish Ministry of Medio Ambiente y Medio Rural y Marino (Exp. 200800050084078) and the E-OBS dataset from the EU-FP6 project ENSEMBLES (http://www.ensembleseu.org) and the data providers in the ECA\&D project (http://eca.knmi.nl). 


\section{References}

Adler, R. F., et al., 2003: The version 2 global precipitation climatology project (GPCP) monthly precipitation analysis (1979-present). J. Hydrometeor., 4, 1147-1167.

Alexandersson, H., 1986: A homogeneity test applied to precipitation data. J. Climatol., 6, 661-675.

Biau, G., E. Zorita, H. von Storch, and H. Wackernagel, 1999: Estimation of precipitation by kriging in the EOF space of the sea level pressure field. J. Climate, 12, 1070-1085.

Chiles, J. and P. Delfiner, 1999: Geostatistics: Modeling Spatial Uncertainty. John Wiley \& Sons.

Deutsch, C. and A. Journel, 1998: GSLIB: Geostatistical Software Library and Users Guide. Oxford Univ. Press,.

Esteban-Parra, M. J., F. S. Rodrigo, and Y. Castro-Díez, 1998: Spatial and temporal patterns of precipitation in Spain for the period 1880-1992. Int. J. Climatol., 18, 15571574.

Fernández, J. and J. Sáenz, 2003: Improved field reconstruction with the analog method: searching the CCA space. Clim. Res., 24, 199-213.

Frei, C. and C. Schär, 1998: A precipitation climatology of the Alps from high-resolution rain-gauge observations. Int. J. Climatol., 18, 873-900.

García-Ortega, E., L. Fita, R. Romero, L. López, C. Ramis, and J. Sánchez, 2007: Numerical simulation and sensitivity study of a severe hail-storm in northeast Spain. Atmos. Res., 83, 225-241, doi:10.1016/j. atmosres.2005.08.004.

Goddard, L., S. Mason, S. E. Zebiak, C. F. Ropelewski, R. Basher, and M. A. Cane, 2001: Current approaches to seasonal-to-interannual climate predictions. Int. J. Climatol., 21, 1111-1152.

Goodess, C. and J. Palutikof, 1996: Development of daily rainfall scenarios for southeast Spain using a circulation-type approach to downscaling. Int. J. Climatol., 18, 1051-1083.

Goodess, C. M. and P. Jones, 2002: Links between circulation and changes in the characteristics of iberian rainfall. Int. J. Climatol., 22 (13), 1593-1615.

Haylock, M., N. Hofstra, A. Klein-Tank, E. J. Klok, P. Jones, and M. New, 2008: A european daily highresolution gridded data set of surface temperature and precipitation for 1950-2006. Journal of Geophysical Research, 113, D20 119.

Hijmans, R., S. Cameron, J. Parra, P. Jones, and A. Jarvis, 2005: Very high resolution interpolated climate surfaces for global land areas. International Journal of Climatology, 25, 1965-1978.

Hofstra, N., M. Haylock, M. New, P. Jones, and C. Frei, 2008: The comparison of six methods for the interpolation of daily european climate data. Journal of Geophysical Research, 113, D21 110, doi:10. 1029/2008JD010100.

Hofstra, N. and M. New, 2008: Spatial variability in correlation decay distance and influence on angulardistance weighting interpolation of daily precipitation over Europe. International Journal of Climatology, 29, $1872-1880$.

Hofstra, N., M. New, and C. McSweeney, 2009: The influence of interpolation and station network density on the distributions and trends of climate variables in gridded daily data. Climate Dynamics, 35, 841 858. doi.10.1007/s00 382-009-0698-1. 
Hutchinson, M., 1998a: Interpolation of rainfall data with thin plate smoothing splines: I two dimensional smoothing of data with short range correlation. Journal of Geographic Information and Decision Analysis, 2(2), 152-167.

Hutchinson, M., 1998b: Interpolation of rainfall data with thin plate smoothing splines: Ii analysis of topographic dependence. Journal of Geographic Information and Decision Analysis, 2(2), 168-185.

Journel, A., 1983: Nonparametric estimation of spatial distributions. Mathematical Geology, 15(3), 445468.

Liebmann, B. and D. Allured, 2005: Daily precipitation grids for South America. Bull. Amer. Meteor. Soc., 86, $1567-1570$.

Matheron, G., 1962: Traite de Geostatistique appliquee. Editions Technip.

Maurer, E. and H. Hidalgo, 2008: Utility of daily vs. monthly large-scale climate data: an intercomparison of two statistical downscaling methods. Hydrology and Earth System Sciences, 12, 551-563.

Maurer, E. P., A. W. Wood, J. C. Adam, D. P. Lettenmaier, and B. Nijssen, 2002: A long-term hydrologically based dataset of land surface fluxes and states for the conterminous United States. J. Climate, 15 (22), $3237-3251$.

Muñoz-Díaz, D. and F. Rodrigo, 2004: Spatio-temporal patterns of seasonal rainfall in Spain (1912-2000) using cluster and principal component analysis: comparison. Ann. Geophys., 22, 1435-1448.

Osborn, T. and M. Hulme, 1997: Development of a relationship between station and grid-box rainday frequencies for climate model evaluation. Journal of Climate, 10, 1885-1908.

Perry, M. and D. Hollis, 2005: The generation of monthly gridded datasets for a range of climatic variables over the UK. Int. J. Climatol., 25 (8), 1041-1054.

Peterson, T., et al., 2008: Homogeneity adjustments of in situ atmospheric climate data: a review. International Journal of Climatology, 18, 1493-1517.

Rodríguez-Puebla, C., A. H. Encinas, S. Nieto, and J. Garmendia, 1998: Spatial and temporal patterns of annual precipitation variability over the Iberian Peninsula. Int. J. Climatol., 18, 299-316.

Rodríguez-Puebla, C., A. H. Encinas, and J. Sáenz, 2001: Winter precipitation over the iberian peninsula and its relationship to circulation indices. Hydrol. Earth Syst. Sc., 5 (2), 233-244.

Sen, C., 2009: Spatial Modeling Principles in Earth Sciences. Springer.

Shepard, D., 1968: A two-dimensional interpolation function for irregularly-spaced data. Proc. 1968 ACM Nat. Conf., ACM, 517-524.

Sillmann, J. and E. Roeckner, 2008: Indices for extreme events in projections of anthropogenic climate change climatic change. J. Geophys. Res., 86, 83-104, doi:10.1007/s10 584-007-9308-6.

Trenberth, K. E., 1997: Short-term climate variations:recent accomplishments and issues for future progress. Bull. Amer. Meteor. Soc., 78, 1081-1096.

von Storch, H., E. Zorita, and U. Cubasch, 1993: Downscaling of global climate change estimates to regional scales: An application to Iberian rainfall in wintertime. J. Climate, 6, 1161-1171. 
Yatagai, A., O. Arakawa, K. Kamiguchi, H. K. abd M.I. Nodzu, and A. Hamada, 2008: A 44-year daily gridded precipitation dataset for Asia based on a dense network of rain gauges. Scientific online letters on the Atmosphere, 5, 137-140, doi:10.2151/sola.2009-035. 